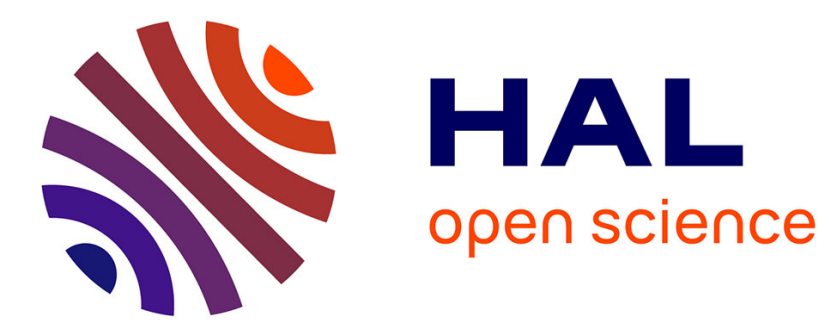

\title{
Tiling an Interval of the Discrete Line
}

Olivier Bodini, Eric Rivals

\section{- To cite this version:}

Olivier Bodini, Eric Rivals. Tiling an Interval of the Discrete Line. CPM: Combinatorial Pattern Matching, Jul 2006, Barcelona, Spain. pp.117-128, 10.1007/11780441_12 . lirmm-00120188

\section{HAL Id: lirmm-00120188 https://hal-lirmm.ccsd.cnrs.fr/lirmm-00120188}

Submitted on 13 Dec 2006

HAL is a multi-disciplinary open access archive for the deposit and dissemination of scientific research documents, whether they are published or not. The documents may come from teaching and research institutions in France or abroad, or from public or private research centers.
L'archive ouverte pluridisciplinaire HAL, est destinée au dépôt et à la diffusion de documents scientifiques de niveau recherche, publiés ou non, émanant des établissements d'enseignement et de recherche français ou étrangers, des laboratoires publics ou privés. 


\title{
Tiling an Interval of the Discrete Line
}

\author{
Olivier Bodini and Eric Rivals \\ L.I.R.M.M. \\ Université de Montpellier II, CNRS U.M.R. 5506 \\ 161 rue Ada, F-34392 Montpellier Cedex 5, France \\ \{rivals, bodini\}@lirmm.fr
}

\begin{abstract}
We consider the problem of tiling a segment $\{0, \ldots, n\}$ of the discrete line. More precisely, we ought to characterize the structure of the patterns that tile a segment and their number. A pattern is a subset of $\mathbb{N}$. A tiling pattern or tile for $\{0, \ldots, n\}$ is a subset $A \in \mathcal{P}(\mathbb{N})$ such that there exists $B \in \mathcal{P}(\mathbb{N})$ and such that the direct sum of $A$ and $B$ equals $\{0, \ldots, n\}$. This problem is related to the difficult question of the decomposition in direct sums of the torus $\mathbb{Z} / n \mathbb{Z}$ (proposed by Minkowski). Using combinatorial and algebraic techniques, we give a new elementary proof of Krasner factorizations. We combinatorially prove that the tiles are direct sums of some arithmetic sequences of specific lengths. Besides, we show there are as many tiles whose smallest tilable segment is $\{0, \ldots, n\}$ as tiles whose smallest tilable segment is $\{0, \ldots, d\}$, for all strict divisors $d$ of $n$. This enables us to exhibit an optimal linear time algorithm to compute for a given pattern the smallest segment that it tiles if any, as well as a recurrence formula for counting the tiles of a segment.
\end{abstract}

\section{Introduction}

Tilings are intriguing in many regards. Their structure, i.e., the way in which the tiles are assembled, may be remarkably complex. As a matter of fact, a theorem from Berger [3] states that, given a set of patterns, determining whether this set tiles the plane by translation is undecidable. This result lets us think there exist sets of tiles that tile the plane only in complex ways. Indeed, Penrose and others [15,5] demonstrated there exist aperiodic sets of tiles (aperiodic means that it tiles the plane, but that none of its tilings admits an invariant by translation). However, some related questions remain open. The smallest known aperiodic set of tiles contains 13 tiles and it is unknown whether there is one with only one non necessarily connected tile. Over and above that, it is undetermined whether the tiling of the plane with one non connected pattern is decidable. Nevertheless, an interesting result from Beauquier-Nivat [1] states that if the pattern is connected the problem is decidable, and if there exists a tiling, there is also a (doubly)-periodic tiling (i.e., one that is invariant by two non-collinear vectors).

Even when restricted to bounded regions of the plane, tiling problems remain difficult combinatoric questions on which little is known. Numerous articles report on specific cases. Among others, the problem of tiling a connected region 
(respectively, a simply connected region) by dominos is polynomial 19 (resp., linear [20]). But, if generalized to a region that is non necessarily simply connected, the problem of tiling by rectangles of size $1 \times 3$ and $3 \times 1$ becomes NP-complete [2].

Regarding these difficulties, it is natural to focus on tiling problems for the discrete line $\mathbb{Z}$. These problems are related to additive number theory, which studies the decompositions of sets of numbers in sums of sets of numbers. A major theorem in this field is the decomposition of integers in sums of 4 squares (Lagrange's theorem), which is written $4 C=\mathbb{N}$ where $C:=\left\{n^{2}: n \in \mathbb{N}\right\}$. Let us also mention Golbach's conjecture (in a letter to L. Euler, 1742), which asks whether any even integer is the sum of two primes $(2 \mathbb{N}=\mathbb{P}+\mathbb{P}$ where $\mathbb{P}$ designs the set of primes).

Indeed in additive number theory, tiling the discrete line with a tile is equivalent, given a set $A$ (representing the tile), to finding a set $B$ (representing the positions of the tile's translations) such that the function $f(a, b):=a+b$ is oneto-one from $A \times B$ into $\mathbb{Z}$. In this case, we denote it $A \oplus B=\mathbb{Z}$. A classical result [12] states that in this case, there always exists a positions set $B$ that is periodic (i.e., for which there is an integer $k$ such that $B+k=B$ ). As an immediate corollary, one obtains the decidability of the tiling of the discrete line by a single pattern. Despite that, its algorithmic complexity remains open although a lot of efforts have been dedicated to study bases for the integers [6, 21]. Moreover, the periodicity of the positions set $B$ raises the question of the characterization of sets $A$ and $B$ such that $A \oplus B=\mathbb{Z} / n \mathbb{Z}$. This problem formulated by Minkowski more than hundred years ago is still mainly unsolved despite the last progresses made by Hajòs $9,10,17,18$.

In this work, we focus on the characterization of sets $A$ and $B$ satisfying $A \oplus B=\llbracket n]$, where $\llbracket n]$ denotes the interval $\{0, \ldots, n-1\}$. This question has been addressed in the literature as the Krasner factorization [11]. Two different constructions of Krasner factorizations have been described in the literature as special cases of Hajós factorizations and used in code theory [13, 7]. In a first part (Sections 2 and 3), we demonstrate using techniques from word theory that if $A \oplus B=\llbracket n \rrbracket$ then either $A$ or $B$ tiles $\llbracket d \rrbracket$, for $d$ a proper divisor of $n$. For any $n \in \mathbb{N}$, let us say a tile is $n$-specific if its smallest tilable segment is $\llbracket n \rrbracket$. More precisely, we exhibit a bijection between $n$-specific tiles and $d$-specific tiles for all strict divisors $d$ of $n$. This result yields a simple formula to count the tilings of $\llbracket n \rrbracket$. The obtained sequence that for each $n$ gives the number of tilings of $\llbracket n \rrbracket$ is described in the Encyclopedia of Integer Sequences [16] (http://www.research.att.com/ $\sim$ njas/sequences/) by Zumkeller without relationship neither to tilings theory, nor to word combinatorics. Besides, we prove a theorem on the size of the smallest tilable segment in function of the tile's diameter. This solves in a specific case a conjecture of Nivat stating that the smallest torus $\mathbb{Z} / n \mathbb{Z}$ that can be tiled by a pattern of diameter $d$ satisfies $n \leq 2 d$. Moreover, we exhibit a linear time algorithm to decide whether a pattern tiles at least one interval of $\mathbb{Z}$ (Section 4). 
In a second part (Section 4), using more algebraic techniques, we demonstrate that any tile of $\llbracket n \rrbracket$ can be decomposed in irreducible tiles (i.e., tiles that are not sums of smaller tiles), which we characterize explicitly. This is in fact a new proof of Krasner factorizations. This combinatorial proof may help developping the theory in the general framework. Furthermore, we know for any $n$ how many irreducible tiles there are. Note that to fit in the page limit, all proofs have been removed from this extended abstract.

\subsection{Definitions and Notation}

Subsets of $\mathbb{N}$ and Polynomials. Let $\mathbb{N}$, resp. $\mathbb{Z}$, be the set of non-negative integers, resp. of integers, and $\mathcal{P}(\mathbb{N})$ the set of finite subsets of $\mathbb{N}$. We denote the set of polynomials with coefficients in $\{0,1\}$ by $\{0,1\}[X]$. We define the mapping $\rho$ that to a finite subset of $\mathbb{N}$ associates a polynomial of $\{0,1\}[X]$ by:

$$
\begin{aligned}
\rho: \mathcal{P}(\mathbb{N}) & \longrightarrow\{0,1\}[X] \\
A & \longrightarrow P_{A}(X):=\sum_{a \in A} X^{a}
\end{aligned}
$$

Clearly, $\rho$ is one-to-one. For all $A \in \mathcal{P}(\mathbb{N})$, we denote by $c(A)$ its minimal element, by $d(A)$ its maximal element, and by \# $(A)$ its cardinality. $d(A)$ is also the degree of $P_{A}$.

Let $A, B \in \mathcal{P}(\mathbb{N})$ and $k \in \mathbb{N}$. The following operations on sets have correspondents for polynomials:

union: $P_{A \cup B}=P_{A}+P_{B}$ if and only if $A \cap B=\emptyset$,

difference: $P_{A \backslash B}=P_{A}-P_{B}$ if and only if $B \subset A$,

translation: if one denotes $A+k:=\{a+k: a \in A\}$, then $P_{A+k}(X)=X^{k} \cdot P_{A}$.

We introduce a notation for the direct sum. Let us denote by $A \uplus B$ the union with repetition for all $b \in B$ of the translates $A+b$. In general, this union is a multi-set on $\mathbb{N}$, i.e., $P_{A \uplus B}:=P_{\uplus_{b \in B} A+b}=\sum_{b \in B} P_{A+b}$ is a polynomial with integral coefficients that are eventually strictly greater than 1 . If there exists $C \in \mathcal{P}(\mathbb{N})$ such that $C=A \uplus B$, then we denote it by $C=A \oplus B$. In this case, $P_{A \uplus B}:=P_{A \oplus B}=P_{A} P_{B}$ and it belongs to $\{0,1\}[X]$. In other words, we investigate the case where the sum is stable in $\mathcal{P}(\mathbb{N})$, or where the product of polynomials is stable in $\{0,1\}[X]$. One says that a polynomial is irreducible in $\{0,1\}[X]$ if it cannot be factorized in $\{0,1\}[X]$. When transposed to subsets of $\mathbb{N}, A$ is irreducible means it is impossible to decompose $A$ in a non trivial direct sum (i.e., other than $\{0\} \oplus A$ ).

Besides, we say $A$ is a prefix of $B$ if and only if $A \subset B$ and $\forall i \in B, i \leq$ $d(A) \Rightarrow i \in A$ (i.e., $B \cap[[d(A)]]=A)$. By convention, one admits that $\emptyset$ is prefix of any other subset of $\mathbb{N}$. We denote by $\llbracket k \rrbracket$ the finite interval of $\mathbb{N}$ of length $k$ whose minimal element is 0 , i.e., the interval $[0, k-1]$. We use the word segment as an alternate for interval.

In the sequel, for any finite subset $A$ of $\mathbb{N}$, we assume that $c(A)=0$ (this is always true up to a translation). We call $A$ a pattern or motif. For a pattern $A$, $d(A)$ is also termed diameter. 


\section{Properties of the Direct Sum}

In this section, we investigate the properties of the direct sum that are useful to study the tilings of an interval. Note that the propositions hereunder are true for subsets of $\mathbb{N}$, but not necessarily for multi-sets on $\mathbb{N}$.

Proposition 1 (Sums of prefixes). Let $A, B, B^{\prime}, C, C^{\prime}$ be subsets of $\mathbb{N}$ such that $A \neq \emptyset$ and $C$ is prefix of $C^{\prime}$. Then, together $A \oplus B=C$ and $A \oplus B^{\prime}=C^{\prime}$ imply that $B$ is prefix of $B^{\prime}$.

Proposition 2 (Sum of a partition). Let $A, B, D$ be subsets of $\mathbb{N}$ such that $D \subseteq A$ and $A \oplus B$ be a subset of $\mathbb{N}$. Let us denote by $\complement_{A} D$ the complement of $D$ in $A$. Then $(D \oplus B)$ and $\left(\complement_{A} D \oplus B\right)$ partition $A \oplus B$.

This proposition is not verified when $A \oplus B$ is multi-set on $\mathbb{N}$ that is not a subset of $\mathbb{N}$. For multi-sets, we have the following property: Let $C, D$ be such that $A=C \biguplus D$, then $(C \oplus B) \biguplus(D \oplus B)=A \oplus B$. In general it is not true that $(C \oplus B) \cap(D \oplus B)=\emptyset$, even if $C \cap D=\emptyset$.

We state two propositions of simplification.

Proposition 3 (Difference of intervals). Let $A, B, C$ be subsets of $\mathbb{N}$ and $m, n \in \mathbb{N}$. If $A \oplus B=\llbracket m \rrbracket$ and $A \oplus C=\llbracket n \rrbracket$ with $n \geq m$, then there exists $D \subset \mathbb{N}$ such that $A \oplus D=\llbracket n-m \rrbracket$ and $D:=\complement_{C} B-m$.

Example 1. Set $A:=\{0,2\}, B:=\{0,1,4,5\}$ and $B^{\prime}:=\{0,1,4,5,8,9\}$. One has $A \oplus B=\llbracket 8 \rrbracket$ and $A \oplus B^{\prime}=\llbracket 12 \rrbracket$, i.e., $m:=8$ and $n:=12$. Let $D:=$ $\complement_{C} B-m=\{8,9\}-8=\{0,1\}$, one obtains $A \oplus D=\llbracket 4 \rrbracket=\llbracket n-m \rrbracket$.

Proposition 4 (gcd of intervals). Let $A, B$ be subsets of $\mathbb{N}$ and $m, n \in \mathbb{N}$. If $M \oplus A=\llbracket n \rrbracket$ and $M \oplus B=\llbracket m \rrbracket$, then there exists $C \in \mathbb{N}$ such that $M \oplus C=$ $\llbracket \operatorname{gcd}(n, m) \rrbracket$.

Proposition 5 (Multiple of an interval). Let $A, B$ be subsets of $\mathbb{N}$ and $n \in \mathbb{N}$ such that $A \oplus B=\llbracket n \rrbracket$. Then, for all $l \in \mathbb{N}, A \oplus\left(\oplus_{i=0}^{l-1}(B+i n)\right)=\llbracket \ln \rrbracket$.

Note that if $\#(A)$ is prime, then $A$ can be decomposed only in the direct sum of the neutral element and itself. We close with an elementary property.

Proposition 6. For any $A \in \mathcal{P}(\mathbb{N})$, one has $\#(A) \leq d(A)+1$ and both members are equal if and only if $A=\llbracket d(A) \rrbracket$.

\section{Tiling an Interval of the Discrete Line}

In this section, let $n \in \mathbb{N}$ be an integer and $f$ be a finite subset of $\mathbb{N}$ such that $d(f)<n$. We use the following notation:

- for any $x<y$, we denote $f \cap[x, y]$ by $f[x, y]$, and $f \cap[x, y[$ by $f[x, y[$;

- for any $0 \leq x \leq d(f)$, let us denote by $f[x]$ the subset $\{i \in f: i<x\}$. 
Definition 1 (Tiling, dual). Let $n \geq 0$ and $f$ be a pattern such that $d(f)<n$. We say that $f$ tiles $[n]$ if and only if there exists $\hat{f}_{n}$, a subset of $\mathbb{N}$, such that $f \oplus \hat{f}_{n}=\llbracket n \rrbracket$. We call $\hat{f}_{n}$ the dual of $f$ for $n$. The element of $\hat{f}_{n}$ are also called the translation positions for $f$.

For a given $n$, the dual is unique. The notion of dual is idempotent: the dual of the dual of $f$ is $f$ itself, and $\hat{f}_{n}$ also tiles $\llbracket n \rrbracket$. We say that a pattern $f$ that tiles $\llbracket n \rrbracket$ is trivial if $f:=[0, n-1]=\llbracket n \rrbracket$ or $f:=\{0\}$. We define a notion of self-period for a pattern. Without loss of generality, we assume that 0 belongs to $f \cap \hat{f}_{n}$ (which is true up to a translation).

Definition 2 (Self-period of a pattern). Let $n \in \mathbb{N}, f$ be a pattern such that $d(f)<n$ and $p$ be an integer such that $0 \leq p \leq d(f)$. We say that $p$ is a self-period of $f$ for length $n$ if and only if for any $i \in[0, n-p[$ one has

$$
i \in f \Leftrightarrow(i+p) \in f .
$$

In other words, $f\left[0, n-p\left[+p=f\left[p, n\left[\right.\right.\right.\right.$. For length $n$, we denote by $\Pi_{n}(f)$ the set of self-periods of $f$, and by $\pi_{n}(f)$ its smallest non null self-period.

Definition 3 (Completely self-periodic). We say that a pattern is completely self-periodic for length $n$ if and only if it is an arithmetic sequence. I.e., if and only if one has $j \in f \Leftrightarrow(\exists i \in[0,\lfloor n / c\rfloor]: j=i c)$, where $c$ denotes the common difference.

Note that if a pattern $f$ is completely self-periodic then its common difference is its smallest non-null period, $\pi_{n}(f)$. We choose the word "self-period" to avoid confusion with the notion of a tiling's period mentioned in the introduction. However, for the sake of simplicity, we use the word period instead of self-period in the sequel, since the context prevents ambiguity. Furthermore, let us point out the connection between the notions of a pattern self-periodicity and of word periodicity.

Example 2. Consider $n:=12$. The pattern $f:=\{0,1,4,5,8,9\}$ has periods 0 , 4, and 8. So, $\pi_{12}(f)=4$ and $\Pi_{12}(f)=\{0,4,8\}$. It can be decomposed in $\{0,1,4,5,8,9\}=\{0,1\} \oplus\{0,4,8\}$. These patterns, $\{0,1\}$ and $\{0,4,8\}$ are completely periodic for lengths 2 and 12 resp., with smallest period 1 and 4 resp.

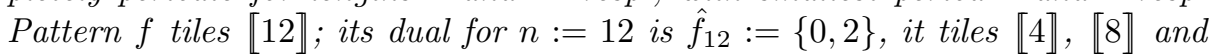
$\llbracket 12 \rrbracket$. It is true that $\#(f) \times \#\left(\hat{f}_{12}\right)=6 \times 2=12$.

\subsection{Properties of Patterns That Tile an Interval}

Let $f$ be a pattern. In the sequel, we assume that $f$ tiles $\llbracket n \rrbracket$. First, we list some elementary properties of $f$.

Proposition 7. Let $f$ be a pattern that tiles $\llbracket n \rrbracket$. First, $\#(f) \times \#\left(\hat{f}_{n}\right)=n$, and second, $d(f)+d\left(\hat{f}_{n}\right)=n-1$. Thus, we have either $d(f)>d\left(\hat{f}_{n}\right)$, or $d(f)<d\left(\hat{f}_{n}\right)$. 
Now, let us state a simple and useful property. It follows from the positivity of the pattern's elements and from the properties of the direct sum.

Proposition 8. For any $x \in \llbracket n \rrbracket$, one has $[0, x] \subseteq f[0, x] \oplus \hat{f}_{n}$.

In a tile, let us call a block a maximal set of consecutive positions. E.g., in $f:=\{0,1,4,5,8,9\}$ the blocks are $\{0,1\},\{4,5\}$, and $\{8,9\}$. A block contains at least an element and may be a singleton.

Now observe the following simple fact: the gap between the first and second block can only be tiled by translations of the first block (and of course of the whole tile). We show below that this implies first, that all blocks have the same length, and second that the first block tiles periodically the interval between 0 and the start position of the second block.

Proposition 9. Let $f$ be a pattern that tiles $\llbracket n \rrbracket$. Assume $f$ comprises $k>1$ blocks; then $f$ is completely specified by the length and starting positions of its $k$ blocks denoted respectively, $\left(b_{i}\right)_{1 \leq i \leq k}$ and $\left(l_{i}\right)_{1 \leq i \leq k}$. W.l.o.g. $b_{1}=0$, and for all $i$ one has $l_{i}>0$. Then:

1. the block length divides $b_{2}$, i.e., $l_{1}$ divides $b_{2}$, and $\hat{f}_{n}\left[b_{2}\right]=\cup_{j=0}^{b_{2} / l_{1}}\left\{j l_{1}\right\}$.

2. all blocks have the same length, i.e., for all $1 \leq i \leq k, l_{i}=l_{1}$.

A corollary of the previous proposition is that the distance between any consecutive block is a multiple of the block length and is larger than $b_{2}$. We can now state a theorem showing that a tile $f$ admits a non null smallest self period.

Theorem 1. A tile $f$ admits a smallest non-null period $\pi_{n}(f)$.

Let us show that the smallest non null period of a non trivial tile is smaller than $\lfloor n / 2\rfloor$. Next proposition demonstrates that this period divides $n$.

Proposition 10. Let $f$ be a pattern that tiles $\llbracket n \rrbracket$ and such that $d(f)>d\left(\hat{f}_{n}\right)$. Then: $\pi_{n}(f) \leq\lfloor n / 2\rfloor$.

Lemma 1. Let $f$ be a pattern that tiles $\llbracket n \rrbracket$ and satisfies $d(f)>d\left(\hat{f}_{n}\right)$. Thus, $\pi_{n}(f)$ divides $n$ and $\left.f\left[\pi_{n}(f)\right] \oplus \hat{f}_{n}=\llbracket \pi_{n}(f)\right]$.

The next corollary follows from the patterns' properties and from Lemma 1.

Corollary 2. If $f$ tiles $\llbracket n \rrbracket$ and $d(f)>d\left(\hat{f}_{n}\right)$ then $d\left(\hat{f}_{n}\right)<\pi_{n}(f)$.

By Proposition 5 , we have that any tile of $\llbracket n \rrbracket$ also tiles $\llbracket l n \rrbracket$ for any integer $l>0$. We deduce the next corollary from Lemma 1 and Proposition 5

Corollary 3. Let $f$ be a pattern and $d$ be the smallest integer such that $f$ tiles $\llbracket d \rrbracket$. If $d>0$, then the $\llbracket l d \rrbracket$, for $l \in \mathbb{N}$, are all the intervals $f$ can tile. 
Theorem 4. Let $n$ be an integer. Among the patterns $f$ that tile $\llbracket n \rrbracket$, it exists a one-to-one mapping that, to any pattern $f$ such that $d(f) \leq n / 2$, associates a pattern that tiles $\llbracket d \rrbracket$ for $d$ a divisor of $n$. This bijection associates to such a pattern $f$ its dual $\hat{f}_{n}$.

One obtains a canonical decomposition of patterns tiling $\llbracket n \rrbracket$ in irreducible patterns. Indeed, Theorem 4 allows us to write any tile $f$ of $\llbracket n \rrbracket$ as the direct sum of i/ a completely periodic pattern for length $n$ (with period a divisor strict of $n$ ) and ii/ one or more patterns that tiles $\llbracket d \rrbracket$, with $d$ a strict divisor of $n$, and are completely periodic for length $d$. This decomposition result is also a corollary of Theorem 7 (section 4).

\subsection{Numbers of Tiles of an Interval}

Let $n \in \mathbb{N}$ such that $n>0$. We denote by $\Xi_{n}$ the set of tiles of $\llbracket n \rrbracket$. Let $\Delta_{n}$ be the subset of patterns in $\Xi_{n}$ whose diameter is smaller than or equal to $\lfloor n / 2\rfloor$ (i.e., those who tile $\llbracket d \rrbracket$ for $d$ a strict divisor of $n$ ), and let $\Psi_{n}$ be the complement of $\Delta_{n}$ in $\Xi_{n}$ (i.e., those patterns with diameter strictly greater than $\lfloor n / 2\rfloor$ ). By definition, one has $\Xi_{n}=\Delta_{n} \cup \Psi_{n}$. We denote the cardinalities of these sets by $\xi_{n}, \delta_{n}$, and $\psi_{n}$, respectively.

Theorem 5. Let $n \in \mathbb{N}$ be an integer such that $n>1$. One has $\xi_{1}=1$ and

$$
\xi_{n}=1+\sum_{d \in \mathbb{N}: d \mid n, d \neq n} \xi_{d} .
$$

Corollary 6. If $n>1$ is prime then $\Delta_{n}=\Psi_{1}, \Psi_{n}=\{\llbracket n \rrbracket\}, \Xi_{n}=\{\{0\}, \llbracket n \rrbracket\}$, $\delta_{n}=\psi_{n}=1$ and $\xi_{n}=2$.

The values of $\xi_{n}$ for $n>0$ are those of Sequence entry A067824 in [16], and (1) corresponds to the recurrence relation given for this sequence by Zumkeller.

Let us denote by $\mu(n)$ the Moebius function. This function satisfies $\mu(n)=$ $(-1)^{r}$ if $n=p_{1} p_{2} \ldots p_{r}$ for distinct primes $p_{j}$ and $\mu(n)=0$ whenever $n$ is divisible by a square. The Moebius inversion states that $f(n)=\sum_{d \mid n} g(d) \Leftrightarrow$ $g(n)=\sum_{d \mid n} \mu\left(\frac{n}{d}\right) f(d)$. Using this property, we can easily obtain the following new induction for $\xi_{n}: \xi_{n}=1-\sum_{d \mid n ; d \neq n} \mu\left(\frac{n}{d}\right)\left(2 \xi_{d}-1\right)$.

\section{Algebraic Approach}

\subsection{Polynomials Decomposition}

Let us denote by $\mathcal{C}$ the set of super-composite integers, i.e., all integers whose prime factorization contains at least two different primes. It is known that $X^{n}-1$ admits a unique decomposition (up to the order of its factors) in irreducible elements of $\mathbb{Z}[X]$. Indeed, $\mathbb{Z}[X]$ is a factorial ring (unique factorization domain). This decomposition is $X^{n}-1=\prod_{d \mid n} \Phi_{d}$, where $\Phi_{d}$ is the $d$-th cyclotomic polynomial [14. We use the following properties of cyclotomic polynomials. 


\section{Proposition 11}

- The degree of $\Phi_{d}$ is $\varphi(d)$, where $\varphi$ is Euler's function.

- $\Phi_{d}(1)=p$ if $d$ is a power of a prime $p$ and $\Phi_{d}(1)=1$ otherwise.

- The polynomial $\Phi_{d}$ belongs to $\{0,1\}[X]$ if and only if $d \notin \mathcal{C}$.

As $\rho$ is a bijection, it induces a one-to-one correspondence between the pairs $(A, B) \in \mathcal{P}(\mathbb{N}) \times \mathcal{P}(\mathbb{N})$ such that $A \oplus B=[n \rrbracket$, and the pairs $(P, Q) \in(\{0,1\}[X] \times$ $\{0,1\}[X])$ such that $P(X) Q(X)=1+\cdots+X^{n-1}$. Moreover, as $1+\cdots+X^{n-1}$ is factorizable in $\prod_{d \mid n, d \neq 1} \Phi_{d}(X)$, there exists a partition of $\{d \mid n, d \neq 1\}$ in $D_{1}$ and $D_{2}$ such that $P(X)=\prod_{d \in D_{1}} \Phi_{d}(X)$ and $Q(X)=\prod_{d \in D_{2}} \Phi_{d}(X)$. Finally, We can notice that if $P$ is in $\{0,1\}[X]$ and $P(X) Q(X)=1+\cdots+X^{n-1}$ then the polynomial $Q$ is not necessarily in $\{0,1\}[X]$. We have the following counterexample : $\left(1+X^{2}+X^{3}+X^{5}\right)\left(1+X-X^{3}+X^{5}+X^{6}\right)=1+\cdots+X^{11-1}$.

\subsection{Results}

Lemma 2. Let $P_{1}, \ldots, P_{k}$ be polynomials of $\{0,1\}[X]$ such that $\prod_{i=1}^{k} P_{i}$ belongs to $\{0,1\}[X]$. For each subsequence $P_{s_{1}}, \ldots, P_{s_{t}}$, with $1 \leq s_{1}, \ldots, s_{t} \leq k$, one has $\prod_{i=1}^{t} P_{s_{i}} \in\{0,1\}[X]$.

For all $n \in \mathbb{N}$, we call total valuation of $n$, denoted by $\nu_{n}$, the sum of the powers in the prime factorization of $n$. We call factorial sequence of $n$, a sequence $u_{0}, u_{1}, \ldots, u_{s}$ such that $u_{0}:=1, u_{s}:=n$, and $u_{i+1} / u_{i}$ is a prime number. Observe that all factorial sequences of $n$ have $\nu_{n}+1$ terms. From a factorial sequence of $n$, we can build a sequence of decomposition $\left(D_{u_{i-1}, u_{i}}\right)_{1 \leq i \leq s}$ with $D_{u_{i-1}, u_{i}}:=$ $\left\{d \mid u_{i}: d \nmid u_{i-1}\right\}$. For conciseness, for all $D \in \mathcal{P}(\mathbb{N})$ we write $\Phi_{D}:=\prod_{d \in D} \Phi_{d}$.

Lemma 3. Let $n, p \in \mathbb{N}$ with $p$ prime. $\Phi_{D_{n, p n}}$ belongs to $\{0,1\}[X]$ and is irreducible in $\{0,1\}[X]$.

Theorem 7 (Krasner Factorizations). Each factorization of $1+\cdots+X^{n-1}$ in irreducible elements in $\{0,1\}[X]$ has the following form $\prod_{1 \leq i \leq s} \Phi_{D_{u_{i-1}, u_{i}}}$ where $\left(D_{u_{i-1}, u_{i}}\right)_{1 \leq i \leq s}$ is a sequence of decomposition of $n$, and reciprocally. Moreover, for all $1 \leq i \leq s, \Phi_{D_{u_{i-1}, u_{i}}}(1)$ is a prime factor of $n$.

Note that the factorization may not be unique.

Example 3. For $n:=12$, the factorial sequences are: $(1,2,4,12),(1,2,6,12)$, and $(1,3,6,12)$. The associated sequences of decomposition are $(\{2\},\{4\},\{3,6$, $12\}),(\{2\},\{3,6\},\{4,12\}),(\{3\},\{2,6\},\{4,12\})$. We obtain that the irreducible factors of $1+\cdots+X^{n-1}$ in $\{0,1\}[X]$ are $\Phi_{2}, \Phi_{3}, \Phi_{4}, \Phi_{3} \Phi_{6}, \Phi_{2} \Phi_{6}, \Phi_{3} \Phi_{6} \Phi_{12}$, $\Phi_{4} \Phi_{12}$.

Theorem 8. The number $v_{n}$ of irreducible factors of $1+\cdots+X^{n-1}$ in $\{0,1\}[X]$ equals $\sum_{d \mid n} \#\{$ prime factors of $d\}$. 
The pattern associated with the polynomial $\Phi_{D_{d, d p}}$ is the arithmetic sequence starting in 0 , of common difference $d$, and having $p$ terms. This gives the precise structure of all tiles of a segment.

A reciprocal polynomial is a polynomial such that $P(X)=X^{n} P(1 / X)$, where $n$ is the degree of $P$.

Corollary 9. Let $f$ be a pattern which tiles an interval. The associated polynomial, $P_{f}$, is reciprocal.

Theorem 10. Let $f$ be a pattern which tiles an interval. Then, the length of the smallest nonempty interval it tiles is smaller than twice the diameter of $f$, i.e., than $2 d(f)$.

Theorem 10 shows that Nivat's conjecture on the upper bound of the tiling periodicity (also mentioned in [4) is true for the special cases considered here.

Theorem 11. Let $f$ be a pattern. Algorithm 1 decides in $O(d(f))$ time whether there is $n \in \mathbb{N}$ such that $f$ tiles $\llbracket n \rrbracket$ and gives the decomposition of $f$ in completely self-periodic tiles.

In Algorithm 1, we use a procedure call CompNextBlock (or CNB for short) that computes all information needed about the next block in the tile. This procedure uses a global variable to scan blocks from left to right in the tile. It stores in a block object the following information: length $(\mathrm{lg})$, distance to previous block or 0 for the first block (dist). Note that the distance is the length of the space that separates two successive blocks. At the last block, dist is set to -1 .

In fact, the algorithm computes the decomposition of the tile in completely self-periodic tiles. It scans the blocks, checks their length, and deduce the level of periodicity they belong to. The information on each level is stored in a Level object: characteristic distance between the last block of previous level and the first block of this level, period, number of repeats, overall length (this is the product of the number of repeats time the period). Other variables are: Lev: table of levels; ilev: index of the current level; pdist: previous distance between two consecutive blocks; fB: first block; nB: current block;

Two cases arise with the distance. Either the distance between the current consecutive blocks is the same as the previous one, then the periodicity remains the same and we have to scan a new copy (repeat) of the current level. Or the distance increases, then the current blocks marks the beginning of a new level of periodicity. Once a periodicity level has been scanned, the variable Lev stores its complete description. A level of periodicity corresponds to a prefix of $f$. To any given level corresponds a prefix of $\hat{f}_{n}$ such that it sums with that level tiles the whole level length. Let us denote that by $\operatorname{Lev}[i] \oplus p_{i}\left(\hat{f}_{n}\right)$, where $p_{i}\left(\hat{f}_{n}\right)$ denotes the prefix of $\hat{f}_{n}$ that tiles Lev[i].lg. The start of a higher level is detected when the inter-block distance increases strictly. The additional space left since the end of the last periodicity level has to be a multiple of Lev[i].lg, such that it can be filled with translates of $\operatorname{Lev}[i] \oplus p_{i}\left(\hat{f}_{n}\right)$. The higher level, say $i+1$ starts necessarily with replicates of all previous periodicity levels. The line "read all previous level" scans these replicates with the descriptions stored in Lev. 


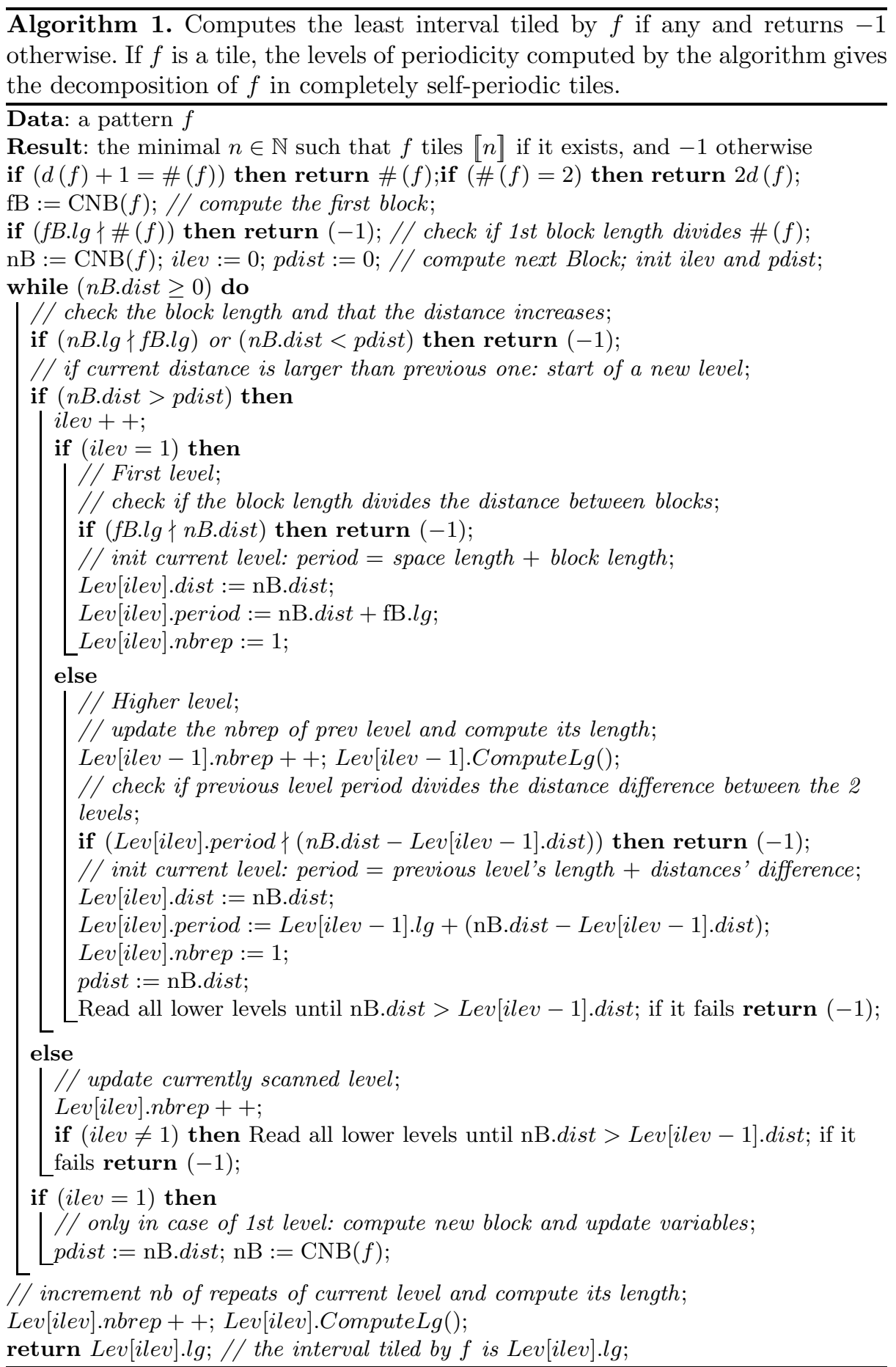


Example 1. Let $f:=[0,6] \cup[21,27] \cup[42,48] \cup[126,132] \cup[147,153] \cup[168,174] \cup$ $[504,510] \cup[525,531] \cup[546,552] \cup[630,636] \cup[651,657] \cup[672,678]$, with $\#(f)=84$ and $d(f)=678$. Then Algorithm 1 will find out that the smallest $n$ such that $f$ tiles $[n \rrbracket$ is $n=1008$ and will decompose $f$ as follows:

$$
\begin{aligned}
f & =[7 \rrbracket \oplus\{0,21,42,126,147,168,504,525,546,630,651,672\} \\
& =[7 \rrbracket \oplus\{0,21,42\} \oplus\{0,126,504,630\} \\
& =[7 \rrbracket \oplus\{0,21,42\} \oplus\{0,126\} \oplus\{0,504\} .
\end{aligned}
$$

The algorithm infers 4 levels of periodicity: the first block $\llbracket 7 \rrbracket$, which has period 1 and is not explicitely store in Lev, and then the following three levels:

$$
\begin{array}{llll}
\text { Lev[1].dist }=14 & \text { Lev[1].period }=21 & \text { Lev[1].nbrep }=3 & \text { Lev[1].lg }=63 \\
\text { Lev[2].dist }=77 & \text { Lev[2].period }=126 & \text { Lev[2].nbrep }=1 & \text { Lev[2].lg }=252 \\
\text { Lev[3].dist }=329 & \text { Lev[3].period }=504 & \text { Lev[3].nbrep }=1 & \text { Lev[3].lg }=1008 .
\end{array}
$$

The dual of $f$ for $n=1008$ is $\hat{f}_{n}=\{0,7,14\} \oplus\{0,63\} \oplus\{0,252\}$, which can also be infered from the length and number of repeats of the periodicity levels.

\section{Conclusion}

This work characterizes the tilings of an interval as direct sums of arithmetic sequences. Counting results obtained also show that, surprisingly, the number of patterns that tile a segment of length $n$ depends, not on the prime factors of $n$, but only on the list of their powers. E.g., segments of respective lengths $n_{1}:=5 \times 7^{2} \times 2^{4}$ and $n_{2}:=13 \times 3^{2} \times 11^{4}\left(n_{1}\right.$ and $n_{2}$ have both $(1,2,4)$ as list of powers), have the same number of tiles. Moreover, for any positive integer $n$ we show that the number of polynomials $p$ with coefficients in $\{0,1\}$ that divide $x^{n}-1$ and such that $\left(x^{n}-1\right) / p(x-1)$ has all coefficients in $\{0,1\}$ equals the number of tiles of $\llbracket n]$. This invalidates the conjecture mentioned in the Encyclopedia of Integer Sequences [16] that these two sequences, A107736 and A067824, are different. Finally, we exhibit a linear time algorithm to recognize a tile and find the smallest $n$ for which it tiles $[n]$. This complexity is otpimal.

The regular structure of the tiles of a segment contrasts sharply with the singular structure of those tiling the torus $\mathbb{Z} / n \mathbb{Z}$. Indeed for this problem, there exists irregular sets $A$ and $B$ such that $A \oplus B=\mathbb{Z} / n \mathbb{Z}[8$. However, our results exhibit a relation between tilings, words and polynomials that opens promising directions for the tiling by a single pattern of the discrete plane or of special cases of the torus. Let us mention that Theorem 7 can easily be extended to higher dimensions. As a matter of fact, one can characterize a pattern that tiles a $d$ dimensional rectangle $n_{1} \times \ldots \times n_{d}$ as the cartesian product of $d$ one-dimensional patterns, each tiling a segment of length $n_{i}$ respectively (with $1 \leq i \leq d$ ). This work also shed light on the complementarity of combinatorial and algebraic approaches for tiling problems.

Acknowledgments. We are grateful to O. Gandouet for reading the manuscript and to F. Philippe for constructive comments. 


\section{References}

1. D. Beauquier and M. Nivat. Tiling the plane with one tile. In Proc. 6th Annual Symposium on Computational Geometry (SGC'90), pages 128-138, Berkeley, CA, 1990. ACM Press.

2. D. Beauquier, M. Nivat, E. Remila, and J.M. Robson. Tiling figures of the plane with two bars. Computational Geometry: Theory and Applications, 5, 1996.

3. R. Berger. The undecidability of the domino problem. Mem. Amer. Math Soc., 66:1-72, 1966.

4. E. M. Coven and A. D. Meyerowitz. Tiling the integers with translates of one finite set. Journal of Algebra, 212:161-174, 1999.

5. K. Culik II and J. Kari. On Aperiodic Sets of Wang Tiles. Lecture Notes in Computer Science, 1337:153-162, 1997.

6. N.G. de Bruijn. On bases for the set of intergers. Publ. Math. Debrecen, 1:232-242, 1950.

7. C. De Felice. An application of Hajós factorizations to variable-length codes. Theoretical Computer Science, 164(1-2):223-252, 1996.

8. L. Fuchs. Abelian Groups. Oxford Univ. Press, 1960.

9. G. Hajós. Sur la factorisation des groupes abéliens. Cas. Mat. Fys., 74(3):157-162, 1950.

10. G. Hajós. Sur le problème de factorisation des groupes cycliques. Acta Math. Acad. Sci. Hung., 1:189-195, 1950.

11. M. Krasner and B. Ranulak. Sur une propriété des polynômes de la division du cercle. Comptes rendus de l'Académie des Sciences Paris, 240:397-399, 1937.

12. J.C. Lagarias and Y. Wang. Tiling the line with translates of one tile. Inventiones Mathematicae, 124(1-3):341-365, 1996.

13. N. H. Lam. Hajós factorizations and completion of codes. Theoretical Computer Science, 182(1-2):245-256, 15 August 1997.

14. S. Lang. Algebraic Number Theory, volume 110 of Graduate Texts in Mathematics. Addison-Wesley Publishing Company, 2nd edition, 2000.

15. R. Penrose. Pentaplexy. Bulletin of the Institute of Mathematics and its Applications, 10:266-271, 1974.

16. N. J. A. Sloane. The On-Line Encyclopedia of Integer Sequences, 2004. Available at http://www.research.att.com/ ${ }^{\sim}$ njas/sequences/

17. S.K. Stein and S. Szabo. Algebra and Tiling: Homomorphisms in the Service of Geometry. Carus Mathematical Monograph 25, MAA, 1994.

18. S. Szabo. Topics in factorization of abelian groups. Birkhauser, 2004.

19. N. Thiant. An $O(n \log n)$-algorithm for finding a domino tiling of a plane picture whose number of holes is bounded. Theorical Computer Sciences, 303(2-3):353-374, 2003.

20. W. P. Thurston. Conway's tiling groups. Am. Math. Monthly, pages 757-773, October 1990.

21. R. Tijdeman. Decomposition of the integers as a direct sum of two subsets. In S. David, editor, Number Theory, pages 261-276. Oxford Univ. Press, 1995. 